(C2020. This manuscript version is made available under the CC-BY-NC-ND 4.0 license http://creativecommons.org/licenses/by-nc-nd/4.0/

\title{
The Role Of Technological Innovation In Plastic Production Within A Circular Economy Framework
}

Roland Yawo Getor ${ }^{\mathrm{a}, 1}$, Nishikant Mishra ${ }^{\mathrm{b}}$, Amar Ramudhin ${ }^{\mathrm{a}}$

a Logistics and Humber Development Institute, University of Hull, Hull, UK. HU6 7RX

${ }^{\mathrm{b}}$ Logistics and Management Systems Department, University of Hull, Hull, UK. HU6 7RX

\section{Abstract}

The growing plastic waste problem has been highlighted by nature programmes like the British Broadcasting Corporation (BBC) War on Plastic series with the World Economic Forum (WEF) 2016 report estimating that by 2050, there could be more plastic than fish in the world's oceans. This has prompted the call for a shift from the linear model of tackling the plastic waste issue to that of a Circular Economy model with increasing demand for recycled resin to be incorporated into the plastic production process alongside virgin resin. However, recycled content are often associated with problems like lack of stability between various combinations of virgin/recycle polymers, which may lead to variations in temperature and inconsistency in the ease of flow attributable to the different temperature and pressure regimes under which virgin raw material and recycled feedstock melt and cool. In this paper, our main contribution is a proposed framework for integrating an enhanced $\mathrm{Al} / \mathrm{DB}$ interface for analysing historical and real time information/data into a joint DSC-TGA system that allows for simultaneous quality control checks and thermal stability tests on different virgin-recycled resin mixing ratios. The database comes equipped with proven estimation methods, testing and statistical analysis results of different resin specimens to serve as a reference point for production engineers in arriving at desired check and test conditions. The information will be particularly useful to plastic product manufacturers when making decisions regarding the right virgin-recycled mix ratio conditions when using different batches of recycled resin or when they switch a supplier.

Key words: Virgin-recycled polymer mix, Circular economy, Intelligent Database, Enhanced AI/DB interface, joint DSC-TGA system

${ }^{1}$ Corresponding author. E-mail address: R.Y.Getor@hull.ac.uk 


\section{Introduction}

The growing magnitude of plastic waste and the sustainable way of handling the waste is currently high on the global and political agenda with increasing pressure for business and Government to work together given its proven negative impact on marine life and the environment. There are various concerns relating to the usage of plastics and disposal of the associated waste including: waste ending up in landfills and in natural habitats; animals ingesting or getting entangled in plastic, and more importantly the non-sustainability of current usage (Thompson et al., 2009). The increase in global plastic production from 1.5 million tonnes in the 1950 s to 335 million tonnes in 2016 has been matched by increased amounts of plastics being discharged into virtually all components of the environment (Alimba and Faggio, 2019). The British Broadcasting Corporation [BBC] 2019 War on Plastics series highlighted the plastic waste epidemic, estimating British households are likely to have 19.5 billion pieces of single-use plastic packaging in their homes. Similarly, the National Geographic estimates that yearly, 8 million tonnes of plastic waste ends up in the oceans and millions of wildlife are killed yearly with nearly 700 species known to have been affected by plastics (Parker, 2019). The World Economic Forum 2016 report on 'The New Plastics Economy' estimates that by 2050, there could be more plastic than fish in the world's oceans. Plastics impose serious problems to living organisms (Chen and Yan, 2019) and microplastics are reported to be present in all components of marine environments including marine organisms (Alimba and Faggio, 2019).

This problem has been attributed to the linear model approach to tackling the plastic waste issue (make, use and dispose). An alternative approach, circular economy framework whereby resources are kept in use for as long as possible, maximum value extracted from them while in use, and product recovery and regeneration at end of life, has gained increasing recognition due to its more sustainable model and resource efficiency prospects (PlasticsEurope 2019). It promotes the responsible 
and cyclical use of resources and includes reducing material input and minimising waste generation to decoupling economic growth from natural resources (Moraga et al., 2019). Thus, the focus here is on reduction, reuse, composting, recycling, energy recovery whiles avoiding or keeping incineration and landfill to the barest minimum. Various ongoing initiatives exist involving: Charities like Ellen MacArthur Foundation and Waste \& Resources Action Programme (WRAP) UK Plastic Pact with businesses from across the entire plastics value chain; Academic/scientific community collaboration like Engineering and Physical Sciences Research Council (EPSRC)/UK Research and Innovation (UKRI) funded UK Universities led circular economy plastic projects (UKRI/EPSRC 2018); Government policy like UK Department for Environment, Food and Rural Affairs [Defra] 2018 Resources and Waste Strategy (RWS) policies including a deposit return scheme (DRS) and UK Treasury plastic tax; and European Union (EU)/International initiatives like European Commission's Circular Economy Strategy on Plastics and Basel Convention Treaty on the movement of plastic waste overseas (UN International Basel Convention Treaty 2018).

Growing environmental concerns associated with the accumulation of plastic waste in the natural environment has led to increasing demand for alternative waste management strategies such as incorporating recycled resin into the plastic production process alongside virgin resin (Payne et al., 2019). However, recycled content are often associated with problems like the ratio of different combinations of virgin/recycle polymers varying from batch to batch which may lead to fluctuations in temperature and inconsistencies in the ease of flow the melted polymer. In addition, there is the increased risk of pollution, which may lead to issues such as variations in the temperature and flow of melted plastics and clogs in the extrusion components. This contamination can originate from foreign material particles that mix with the product during recycling or from different plastic varieties that are added during the recycling but that are difficult to identify. Recycled feedstock may result in products that suffer from material variability because it is an extremely difficult task achieving consistency in all batches making them strong today and weak tomorrow. Ultimately, the end use of the produced part will motivate the choice of material as 
well as choice of recycled versus virgin plastic. In view of these issues, the plastic manufacturer and the industry in general have to perform several experiments and tests to determine the ideal conditions like quality standard, temperature and melt flow range needed to achieve the required product consistency.

The plastic production process described above using virgin-recycled polymer mix is time consuming, resource intensive and uses minimal amount of recycled content due to issues surrounding consistency, variability and contamination discussed. Hence, the motivation of this article to examine how Artificial Intelligence technology can be introduced into the plastic production process to make it smoother, less complicated while ensuring circular economy is promoted through the incorporation of more recycled content. Industry 4.0 has opened up avenues by transforming manufacturing through the integration of Artificial Intelligence (Al) and Database (DB) technologies with thermal analysis techniques (Differential Scanning Calorimetry (DSC) and Thermal gravimetric analysis (TGA)) into the plastic production process.

In this paper, our main contribution is a proposed framework that integrates an enhanced $\mathrm{Al} / \mathrm{DB}$ interface with both historical and real-time data/information processing capability into a joint DSC-TGA system for simultaneous quality control checks and stability tests to be carried out during the plastics production process using different virgin-recycled resin mix ratios. The database comes equipped with proven estimation methods based on analysis of thermal and mechanical properties and structure (morphology) of different resin specimens to serve as a reference point for production engineers in arriving at desired check and test conditions using the DSC-TGA software. In addition, it will enable production engineers to continuously carry out quality control/quality assurance checks and monitor the temperature and chemical composition of the polymer (Majewsky et al., 2016). The information provided by the database will also be particularly useful to plastic product manufacturers when making decisions regarding the right virgin-recycled mix ratios conditions when using different batches of recycled resin or when they switch a supplier. This is explained further in the main article. The rest of the paper is organised as follows: section 2 focuses on review of literature whereas section 3 
gives an overview of plastics (classification [3.1], rationale and implications of adding recycled content [3.2], plastics production process using virgin raw materials only [3.3] and a combination of virgin-recycled raw materials [3.4]). The concluding section (section 4) outlines the main contributions [4.1], managerial implications [4.2] and limitations and future outlook [4.3] of the study.

\section{Literature review}

We draw on literature on circular economy relating to plastic waste and on thermal analysis techniques like DSC and TGA used in quality control/quality assurance checks and material development of plastic materials and components to discuss work being done and its contribution to the ongoing debate in the plastics area. This covers the role of database tools like Identify evaluation software within a joint DSC/TGA system in enhancing quality control checks and stability tests. We expand this further by focusing on the potential of $\mathrm{Al}$ in boosting information/data processing within fields like computer science, nature, education and gaming. This is adapted to the production of plastics by proposing an intelligent database interface framework with capability for processing historical and real-time information within a circular economy environment.

\subsection{Circular economy approach to managing plastic waste}

The importance of circular economy approach to dealing with the current plastic waste epidemic is important as it is restorative and regenerative and as such allows materials to flow constantly around a closed loop/sustainable system. Thus, this departs from the 'take-make-dispose' model of using the material once and disposing of it, which used to characterise the treatment of plastic waste. Charles et al. (2018) argue in favour of this approach noting retention of materials within the economy through recovery and regeneration of products at the end of each service life maximises their economic productivity, offsets demand for primary resources and decouples growth from resource consumption. Similarly, Circular Economy is high on the political agenda and especially in Europe, expected to promote economic growth by creating new businesses and job opportunities, improving security of supply while 
at the same time reducing environmental pressures and impacts (Kalmykova, et al., 2018). This is especially important in meeting the United Nation (UN) 2012 Sustainable Development Goal on Sustainable Consumption and Production, of substantially reducing waste generation through prevention, reduction, recycling and reuse (United Nations [UN] 2012). Likewise, it is crucial to reducing the loss to the global economy with the World Eonomic Forum estimating plastic packaging waste accounts for $£ 80-£ 120$ billion annual loss (Opsomer and Pennington, 2016).

At the global level, 'The New Plastics Economy Global Commitment' led by the Ellen MacArthur Foundation in collaboration with the UN Environment Programme has as its vision, a circular economy for plastics in which it never becomes waste and have three actions to:

- 'eliminate' all problematic and unnecessary plastic items;

- 'innovate' to ensure plastic needed are $100 \%$ reusable, recyclable or compostable and

- 'circulate' plastic items to keep them in the economy/environment) to realise that goal.

It unites businesses, governments and other organisations behind the vision with $20 \%$ of the signatories being companies of all plastic packaging produced globally (Ellen MacArthur Foundation, 2017). The European Commission also adopted the 'European Strategy for Plastics in a Circular Economy' in 2018 with innovation being the key driver. They aim to modernise the industry, improve plastics and plastic products design, to boost reuse and recycling as well as demand for recycled plastics (European Commission [EC] 2018). Similarly, the 2018 UK Plastics Pact led by WRAP and supported by Defra aims to achieve by 2025 , the overall goal of zero plastic packaging leaked into the environment by meeting these targets:

- Eliminate problematic or unnecessary single-use packaging through redesign, innovation or alternative (reuse) delivery models

- $100 \%$ of plastic packaging to be reusable, recyclable or compostable

- $70 \%$ of plastic packaging effectively recycled or composted

- $30 \%$ average recycled content across all plastic packaging 
With 18 months into the seven year initiative, the UK Plastics Pact has over 120 businesses (including the big 6 supermarkets), governments, local authorities and organisations committed to tackling plastic pollution by transforming the way we make, use and dispose of plastic packaging in the UK (WRAP, 2018).

Calleja (2019) argues that the 'New Plastics Economy' must be a circular economy, because it is about retaining the value of materials in the economy and as such this approach is justified due to the unique characteristics of plastics and the urgent need for an approach that integrates all parts of its cycle. He notes it must be one that eliminates waste, maximises value, uses plastic efficiently and in so doing help protect our environment, reduce marine litter, greenhouse gas emissions and dependence on imported fossil fuels. Mrowiec (2018) is also of the view that changes to be introduced in design and production of plastics will contribute to increasing their recycling rates and will help achieve the European Union (EU) priority set for an energy union with a modern, low carbon and energy efficient economy.

\subsection{Thermal analysis techniques and online database search capabilities}

Identification of materials such as plastics is of immense importance especially with regard to the measures put in place to maintain the quality of the raw materials and the tests carried out to identify potential problems with the components of the product (Schindler et al., 2017). Analytical techniques like Differential Scanning Calorimetry (DSC) and Thermo Gravimetric Analysis (TGA) are commonly used in this regard to study plastic polymers. For instance, a DSC instrument is useful in controlling the quality of recycled granule feedstock due to its ability to monitor and detect impurities in a rapid and cost-effective manner. TGA is also able to monitor the stability of the plastic as it undergoes transition from pellets to molten and back to solid form by monitoring the mass as temperature changes.

Lowe (2017) acknowledges the increasing popularity of plastics and polymer based materials and notes that they continuously find their way into new and more demanding applications because of their excellent mechanical performance, 
physical/thermal properties and light weight characteristics. However, she observes that to achieve optimum performance and properties for a particular application, additives and fibres are routinely incorporated into the plastics. Therefore, she argues extensive analysis and characterisation is required using techniques like DSC and TGA to achieve the desired properties and continuously monitor the quality of the product (Lowe, 2017). In addition to giving insight into the thermal properties of polymer materials and products, thermal analysis techniques also gain information regarding the condition or processing history of specific samples relative to reference samples. Netzsch Group 2019 notes this makes thermal analysis well suited to quality control and assurance allowing incoming materials to be inspected and selected easily and safely. The leading instrumentation specialist company argues DSC and TGA analysis, in accordance with International Organisation for Standardisation (ISO) 11357 standard, provides valuable information (Product identification and characterisation, Advanced material analysis and process/product information) for development and quality control of polymers and on general principles of thermogravimetry for polymers.

Differential Scanning Calorimetry (DSC) monitors the difference in temperature between the sample and a reference material against time or temperature in a specified atmosphere. The flow of heat in and out of a sample and a reference material is measured as a function of temperature as the sample is heated, cooled or held isothermally at a constant temperature (Fortunato, 2013; Dwiverdi et al., 2017). The literature notes DSC is widely used in examining polymeric materials to determine their thermal transitions (glass transition, crystallisation and melting temperature) with the observed transitions useful in comparing materials. DSC has the capability to detect endothermic (absorption of heat) and exothermic (release of heat) effects, determine peak areas (transition and reaction enthalpies), determine temperatures that characterise a peak or other effects and measure specific heat capacity (Fortunato, 2013). It is seen as an effective analytical tool to characterise properties of a sample like polymer with the result of a DSC experiment depicted by a curve of heat flow against temperature (Schick, 2009). Fortunato (2013) observes the measurement signal is the energy absorbed or released by the sample in 
milliwatts. Similarly, he notes different measurement principles, sensors, signal processing accessories and evaluation capabilities make DSC one of the most common and versatile techniques in material characterisation.

Thermal gravimetric analysis (TGA) is a thermal analysis technique used to measure the mass of a sample over time as temperature changes. This procedure provides information about physical phenomena (example, phase transitions and absorption), chemical phenomena (including thermal decomposition) and solid-gas reactions (example, oxidation and reduction). This analysis is carried out with the aid of an instrument known as thermogravimetric analyser which measures continuously mass while temperature of a sample (for instance, polymer) is changed over time. A typical instrument consists of a pan resting on a sensitive analytical balance with the test sample placed on the pan and heated externally (Basu, 2018). The literature notes it can be used for characterising materials by analysing the characteristic decomposition patterns and in that regard, a very useful technique for studying polymeric materials such as thermoplastics, thermosets, plastic films, fibers and fuels. Since polymers melt before they decompose, TGA is useful in investigating the thermal stability of polymers as most of them melt or degrade before $200^{\circ} \mathrm{C}$. However, TGA is also suitable for the special class of thermally stable polymers that are able to withstand temperatures of at least $300^{\circ} \mathrm{C}$ in air and $500^{\circ} \mathrm{C}$ in inert gases without structural changes or strength loss (Liu and Yu, 2006).

Netzsch 2019 note TGA provides complementary and supplementary characterisation information to DSC. The simultaneous application of TGA and DSC to the same sample in a single instrument has the main advantage of identical test conditions for signals (such as same atmosphere, glass flow and heating rates, radiation effects, vapour pressure of the sample). Schindler et al. (2017) acknowledge the potential of simultaneously undertaking a search of DSC and TGA signals in a database. TGA measurement information include mass changes, temperature stability, filler content, compositional analysis of multi-component materials whereas DSC measurement information captures specific heat capacity, 
melting/crystallisation behaviour, solid-solid transitions, purity determination for additives and cross-linking reactions.

The literature argues that the added advantage of thermal analysis techniques like DSC is the ability to incorporate a computer-based system that allows for a measurement to be identified by comparing it with a database entry. This became a reality in 2014 with the introduction of Identify, the first computer-based tool with graph recognition and database capability, as part of NETZSCH Proteus $®$ evaluation software (Schindler et al., 2016). The added advantage of this online tool is that it helps overcome the limitation of comparing own data with printed collection of measurement results like "Atlas of thermoanalytical curves" and NETZSCH's "annual for science and industry, volume series" (Fueglein, 2015). It has the ability to conduct software-based library comparisons of polymer samples like identification of pure substances and mixtures (Fuelglein and Kaisersberger, 2015). In addition to one-to-one comparison between a user defined measurement for a database search and database entries, simultaneously there is also the possibility of the user to use their own criteria to classify and for that matter, define quality control checks and failure analysis tests. For example, DSC based studies undertaken by Schindler in 2014 and 2016 show Polyamide (PA) can routinely be differented from Polyethylene and from combinations of the two. Identify also allows for extension to other measurement techniques like inclusion of data from TGA into the same database with databases like that of NETZSCH supporting over 1000 entries from various fields including polymers (Schindler et al., 2016).

Another feature of Identify is that it can be used to archive information from literature and graphs. For instance, it allows information to be incorporated into one or more libraries and specific information (graphs and literature) can be retrieved from the database through, say, alphabetical filtering (Schindler et al., 2016). The authors note that the ability to search and find database entries is very useful for a priori information gathering about the behaviour and expected measurement results of a sample before actual measurement is carried out. Similarly, it provides an avenue for 
gaining adequate insight from the database as to the conditions that need to be satisfied before carrying out a measurement.

The literature argues that whiles Identify can recognise measurement curves or select sections of it through comparison with information stored in the database, it should be noted that recognition of a substance does not automatically translate into identification. Accordingly, (Schindler et al., 2016) point out that it is not always possible to completely identify materials due to issues like having different interpretations for a measurement, dependence on the measurement conditions and lack of similar reference information or data in the database. A general limitation of database search routines is being confronted with several possible meanings for a measurement curve. For instance, identification based on the specific heat capacity or the reversible thermal response of the material to glass transition, crystallisation or melting is not a unique way of identifying a material (Schindler et al., 2016; 2017). Similarly, another common obstacle of relying on identification of materials through thermal analysis is that the measurement outputs (curves) may be susceptible to the conditions existing at the time of measurement. This could result in DSC graphs based on the same material yielding a similarity value of less than $100 \%$ because of differences in the heating rates and sample masses used. A further limitation is where a search outcome would have yielded a positive outcome but the database has not yet been populated with comparative information. Where such a situation arises, the user can put together their own bespoke application database for future measurement purposes and also for comparing new material measurement results with past stored database measurements on a material.

\subsection{Integration of $\mathrm{Al}$ and database into thermal analysis techniques}

An important step in promoting recycling as a key tool in the circular economy model is ensuring the recycled raw material and final output produced from it are of high quality. This is important if product manufacturers are to come on board fully by moving away from virgin materials. The fourth industrial revolution has opened up avenues like industry 4.0 which is transforming manufacturing through digitisation regarding how things are produced with the trend towards automation and data 
exchange in manufacturing technologies and processes including Artificial Intelligence $(\mathrm{Al})$. Digitalisation of the production process helps to implement the assessment tools and the decision making systems businesses need to have in place to achieve the desired performance indicators for measuring progress in a sustainable way (García-Muiña et al., 2018). Data and analytics are seen as being at the core capabilities with a key driver being digitisation and integration of vertical and horizontal value chains (Geissbauer, 2016). The growth of material database together with the advancements in data science and $\mathrm{Al}$, and the innovations brought by computer programs like AlphaGo (Silver et al., 2016) has ushered in a new age termed the "fourth paradigm of science" (Agrawal and Choudhary, 2016) or the "fourth industrial revolution" (Gil et al., 2014). In view of progress made in computational technologies, great strides have been made in the field of material design through modelling and simulation which has proved useful in the prediction of material properties especially in the absence of analytics (Chen et al., 2020). In this regard, current innovations in technology have ensured Al can be incorporated into database management systems to form intelligent databases. Hence, computer based systems incorporating intelligent databases with capability for storing, processing and analysing polymer related data can be combined with thermal stability techniques for quality control/assurance checks of the raw material and stability tests of the components of the final output during the production process.

Artificial intelligence is the art of creating intelligence in machines and defined as the branch of computer science that study and develop intelligent machines and softwares (Bhatia et al., 2013). It aims to equip software with the ability to analyse its environment based on predetermined rules and search algorithms or pattern recognising machine learning models and making decisions off those analyses. This has been defined by key Al researchers as the study and design of intelligent agents, that is, systems that perceive their environment and take action to maximise their chances of success, the ultimate goal being to make computer programs with the capability of solving problems and achieving goals in the world (Bhatia et al., 2013). The literature acknowledges there are four distinct categories of Al (Reactive, Limited Memory, Theory of Mind and Self-Aware Al) but in practical terms, it can be 
used in commercial or consumer settings for speech recognition, natural language processing or computer vision. Since the focus of this paper is on identification and prediction we will be concentrating on pattern recognition and timeline capability. For instance, it has been portrayed as computing systems with the ability to engage in human-like processes like learning, adapting, synthesizing, self-correction and use of data for complex processing tasks (Popenici and Kerr, 2017). Machine learning has great potential in accelerating the discovery of innovative materials (Wu et al., 2019). The concept of machine intelligence has been used in various fields such as computer science for image recognition (He et al., 2016), nature (Silver et al., 2017) and gaming (Brown and Sandholm, 2017) to train and manipulate massive amounts of data to match or outperform humans in challenging tasks. Through the inspiration of machine-learning-assisted polymer, technology has also been used to demonstrate the successful discovery of new polymers using thermal conductivity (Wu et al., 2019). This discovery was made possible through the interaction between machine intelligence trained on a substantially limited amount of polymetric properties data, expertise from laboratory synthesis and advanced technologies of thermophysical property measurements.

A database is an electronic filing system with all the physical data transformed into records and stored for future use. There are different types of database including relational and cloud databases and contain both present as well as historic data thereby providing efficient and fluent use of stored information for a variety of applications (Bhatia et al., 2013). They facilitate the storage, manipulation and retrieval of data under efficient information management systems (DBMS) resulting in effective task allocation and increase in productivity (Nihalani et al., 2009). The literature notes database systems are important in the management of data and with advancement in web technology, they have become repository of large amounts of data (Ma, 2007). It is therefore vital to utilise such vital data in a way that maximises the returns for decision making purposes. Data within databases is represented by applications of simplified concepts which are used in storing large volumes of the data and also in retrieving similar amounts of the same data using the right programme query (Bertino et al., 2001). Knowledge-based systems (KBS) are those 
that generate, extract and utilise useful knowledge from different sources, data and information already present and inputted in a database (Bhatia et al., 2013). They are capable of making decisions based on the knowledge within them and as such understand the context of the data being processed. It has an interface engine that serves as the search engine and knowledge base that acts as the repository for knowledge. Relational database management systems (RDBMS) are those tailored to addressing specific problem types, that is, storing, processing and retrieving large volumes of data of the same type (Bhatia et al., 2013). The literature notes that materials design approaches that are driven by data have been described as the "fourth pillar" in scientific research and to that effect, many information driven innovations like the de novo design of polymers including frameworks like material informatics (Rajan, 2013; Sarkisov and Kim, 2015) and polymer informatics (Adam, 2010; Audus and de Pablo, 2017).

Integration of Artificial Intelligence and Database Management Systems (DBMS) is crucial for next generation computing and continued development of DBMS technology (Nihalani et al., 2009). It is crucial for the continued development of DBMS technology and the effective application of significant Al technology. The idea of intelligent databases combines databases (Knowledge Based Systems [KBS] and Relational Database Management Systems [RDBMS]) and artificial intelligence to store, process and analyse data (Parsaye et al., 1989; Bhatia et al., 2013). This represents an extension to the traditional databases, which are user interactive and apply artificial intelligence at three levels of database intelligence [high-level tools, user-interface and underlying database engine] (Nihalani et al., 2009). Since both artificial intelligence and database management systems represent well developed technologies, a case can be made for their integration. This has been necessitated by: access to huge volumes of shared data for information processing; managing data and information efficiently and intelligently manipulating data (Bhatia et al., 2013). Chen et al. (2020) acknowledge that advancement in computer resources for undertaking computational task, Al and the increase in material based databases has made it possible for ML-assisted materials design tools to quantitatively predict the structure property of materials more efficiently and accurately. The literature 
identifies four approaches for integrating $\mathrm{Al}$ and database (DB) through an intelligent database interface (IDI) namely: extending Al system to DB; extending DB system to $\mathrm{Al}$; loose coupling between $\mathrm{Al} / \mathrm{DB}$ and enhanced interface between $\mathrm{Al} / \mathrm{DB}$ (Bhatia et al., 2013). An enhanced interface between $A \mathrm{l} / \mathrm{DB}$ is the most advanced and preferred because in addition to the substantial enhancement over the other IDI systems, it provides a more powerful and efficient interface between the two systems (Al/DB). This method of Al/DB integration allows the system to make use of existing $\mathrm{Al}$ and DB technologies as well as future innovations in both. Any performance and under-utilisation issues associated with DBMS by the Al system are handled with varying degrees of success, firstly by increasing the functionality of the interface itself and if necessary, enhancing either the Al system or the DBMS (Bhatia et al., 2013) .

\subsection{Identification of the literature gap}

We have demonstrated the use of DSC-TGA techniques in the identification and measurement of specific polymers during the plastic production process using a database evaluation software like Identify. Even though this process incorporates the use of database, from a circular economy perspective this does not represent efficient and optimal use of resources as the software supports minimal recycled polymer content (about 10\%) due to consistency and variability challenges surrounding the use of recycled polymers. We have also showed how Al has been effectively employed in fields like computer science, nature and education using computational technologies, modelling and simulation to undertake polymer based quality control measurements and to predict material properties of polymers. In this paper, we merge thermal analysis techniques and Al to propose an intelligent database interface framework that promotes circular economy and improves efficiency by departing from $100 \%$ virgin material usage to one incorporating increased proportions of recycled content in the plastics production process. The enhanced $\mathrm{Al} / \mathrm{DB}$ interface has capability for processing both historical and real time data/information and forecasting the future horizon of companies with significant success rate by using adequate visualisation and analytic tools to gain valuable insights. For instance, it provides a plastic production engineer a yardstick to 
undertake quality control and stability checks on an ongoing basis during the polymer production process using similar predefined and real time measurement/identification criteria in the AI/DB system.

\section{Overview of plastics}

Plastics can be defined as 'a group of organic polymers, including synthetic, semi-synthetic or natural materials that are malleable and can be molded into solid 
objects' (Chen and Yan, 2019). Similarly, American Chemistry Council notes that plastics, also known as polymers, are produced by the conversion of natural products or by the synthesis from primary chemicals generally originating from oil, natural gas or coal. Naturally occurring polymers include tar, cellulose, tortoiseshell and latex from tree sap whereas synthetic polymers include polyethylene (used in plastic bags), polystyrene (used in making Styrofoam cups), polyvinyl chloride (used for food wrap, bottles, and drain pipe) and Teflon (used for nonstick surfaces).

\subsection{Classification of plastics}

The literature notes that plastics are produced through two main processes, polymerisation and polycondensation, both of which require specific catalysts. There are different types of plastics and different ways of classifying them. For instance, they can be grouped into two main polymer families: thermoplastics and thermosets (PlasticsEurope 2019). Thermoplastics are plastics, which soften when heated and once cooled, retain their shapes and forms. The process is reversible and can be performed several times, where the plastic can be remelted and reformed. It can also be moulded into complex shapes (Sastri, 2010). Thermosets on the other hand, strengthens when heated, but cannot be remoulded or heated after the initial forming does not soften once moulded. Examples of each are presented in Table 1

Table 1. Types of plastics by main polymer group

\begin{tabular}{|c|c|}
\hline Thermoplastics & Thermosets \\
\hline Acrylonitrile butadiene styrene (ABS) & Epoxide (EP) \\
\hline Polycarbonate (PC) & Phenol-formaldehyde (PF) \\
\hline Polyethylene (PE) & Polyurethane (PUR) \\
\hline Polyethylene terephthalate (PET) & Unsaturated polyester resins (UP) \\
\hline Polytetrafluoroethylene (PTFE) & \\
\hline Polyvinyl chloride (PVC) & \\
\hline Polymethyl methacrylate (PMMA) & \\
\hline Polypropylene (PP) & \\
\hline Polystyrene (PS) & \\
\hline Expanded Polystyrene (EPS) & \\
\hline
\end{tabular}

Source: PlasticsEurope 2019

Thermoplastics can further classified based on their performance characteristics (commodity, engineering and high performance) and whether they can be structured 
(crystalline) or not (amorphous) as captured in Figure 1. Commodity plastics account for about $80 \%$ of plastics used for medical devices whereas engineering plastics will have a higher rate of growth due to demand for them in surgical, diagnostic testing, drug delivery and preventive medicine. High-performance engineering materials are also used in implants and high-performance analytical and diagnostic instrumentation (Sastri, 2010; Gehring, 2000).

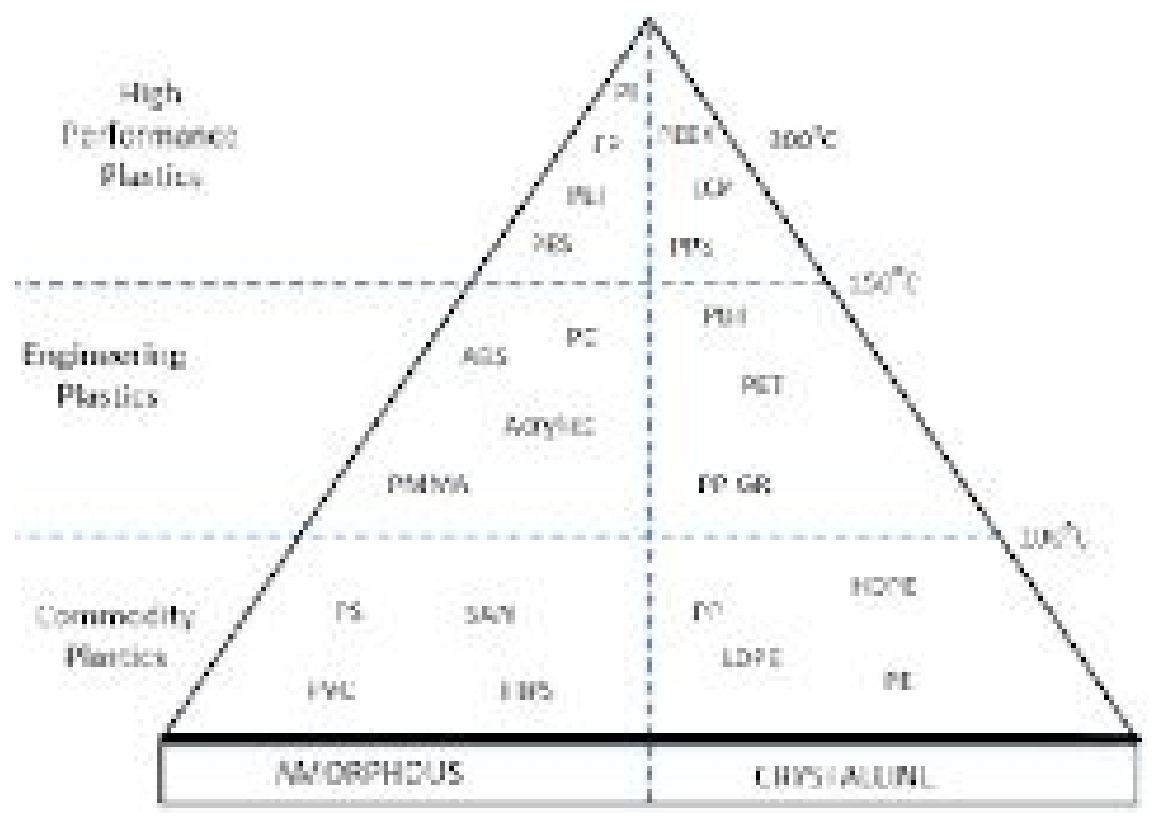

Figure 1. Classification of thermoplastics Source: Gehring (2000); Sastri (2010)

Another way of classifying plastics is based on specific class numbers. In this regard, in 1988 a classification system was established by the Society of the Plastics Industries (SPI) (now known as Plastics Industry Association, [PLASTICS]) to assist consumers and manufacturers to properly recycle items (Table 2). Under this classification system, each plastic product is given a SPI number, usually at the bottom of the product to distinguish different materials of the plastics. 
Table 2. Society of the Plastics Industry (SPI) classification of plastics for consumers and recyclers

\begin{tabular}{|c|c|}
\hline TYPE OF PLASTIC & COMMON HOUSEHOLD USES \\
\hline $\begin{array}{l}\text { Polyethylene Terephthalate } \\
\text { Sometimes absorb odours and flavours from food and } \\
\text { drinks stored in them. }\end{array}$ & $\begin{array}{l}\text { PET(E) Commonly recycled } \\
\text { Mineral Water; Fizzy drink and beer bottles; Pre-1 } \\
\text { bags; } \\
\text { Boil in the bag food pouches; Soft drink and water bo }\end{array}$ \\
\hline $\begin{array}{l}\text { High-Density Polyethylene } 2 \\
\text { Very safe and not known to transmit any chemicals } \\
\text { into foods or drinks }\end{array}$ & $\begin{array}{l}\text { HDPE Commonly recycled } \\
\text { Detergent, bleach and fabric conditioner bottles; S } \\
\text { liners; Milk and non-carbonated drinks bottles; Toy } \\
\text { Plant pots }\end{array}$ \\
\hline $\begin{array}{l}\text { Polyvinyl Chloride } \\
\text { Can be harmful if ingested, should not come in } \\
\text { contact with food items }\end{array}$ & $\begin{array}{l}\text { PVC Sometimes recycled } \\
\text { Credit cards; Carpet backing and other floor cove } \\
\text { Guttering Pipes and fittings; Wire and cable sheathin }\end{array}$ \\
\hline $\begin{array}{l}\text { Low-Density Polyethylene } \\
\text { Very healthy plastic that tends to be durable and } \\
\text { flexible }\end{array}$ & $\begin{array}{l}\text { LDPE Sometimes recycled } \\
\text { Cling-films; fertiliser bags; refuse sacks; Packagir } \\
\text { bottles; Irrigation pipes; Thick shopping bags (cloth } \\
\text { applications; Some bottle tops }\end{array}$ \\
\hline Polypropylene & $\begin{array}{l}\text { PP Occasionally recycled } \\
\text { Most bottle tops; Ketchup and syrup bottles; Yoghur } \\
\text { Potato crisp bags; Biscuit wrappers; Crates; Plant Pot } \\
\text { boxes; Heavy duty bags/tarpaulins }\end{array}$ \\
\hline Polystyrene & $\begin{array}{l}\text { PS Commonly recycled (difficult to do) } \\
\text { Yoghurt containers; egg boxes; Fast food trays; } \\
\text { disposable cutlery; Seed trays; Coat hangers; Low cos }\end{array}$ \\
\hline
\end{tabular}




\begin{tabular}{|l|l|}
\hline Code 7 (includes Polycarbonate and Polylactide) & $\begin{array}{l}\text { OTHER (Difficult to recycle) } \\
\text { Nylon (PA); Acrylonitrile butadiene styrene (ABS); } \\
\text { polymers. } \\
\text { (Have a wide range of uses, particularly in engineerir }\end{array}$ \\
$\begin{array}{l}\text { Used to designate miscellaneous types of plastic not } \\
\text { covered by the other six codes }\end{array}$ & \\
\hline
\end{tabular}

Source: Plastics Industry Association [PLASTICS] 1988 


\subsection{Plastics Production process using virgin polymers}

The stages for the production of a virgin plastic like PET are discussed from the Raw material to the final output stage as shown in Figure 2. The process starts with the sourcing of primary plastics raw materials in the form of pellets. They are produced from derivatives of crude oil (ethane and propane) that are mixed together under high temperature to produce Ethylene and Propylene. These are combined with catalyst in a reactor to produce fluff, which is further combined with additives in a blender and melted in an extruder and transformed into polymer pellets (Wong, 2010). The pellets once acquired by the plastics manufacturer is dried in a dehumidifying dryer to remove any moisture to at least $0.005 \%$ for up to about $4 \mathrm{hrs}$ at about $160^{\circ} \mathrm{C}$ (see Figure 2).

The plastic pellets are then injected into a machine, injection moulder for the next stage of the production process. The process takes up to about 2 minutes and the machine is made up of three constituent parts: the clamping, injection and moulding units. The clamping unit's role is to hold the two halves of the injection mould together during the production process. The injection phase starts with the pellets loaded into the injection unit, through a hopper situated at the top, which feeds into a cylinder where the pellets are heated until they become molten. A motorised screw then mixes the molten pellets, forcing them to accumulate at the front of the screw where the injection process starts. The molten plastic is then inserted into the mould through a sprue or channel with the pressure and speed controlled by the screw. A pause in the injection process ensures pressure is applied to the molten plastic injected into the mould till all the mould cavities are filled. The melted, liquid plastic is injected into a tightly closed, chilled mould where water cooler containing chilled water $\left(15^{\circ} \mathrm{C}-20^{\circ} \mathrm{C}\right)$ with pressure (5Bar) is applied to cool the molten plastic into a solid preform within the mould. Finally, the clamping unit is opened separating the two halves of the mould and the preform ejected mechanically or by compressed air (Patterson and Rothwell, 2019; Sciencing.com, 2018).

In a two stage production process, the preform is transferred into a blow moulding machine. The preform is clamped into a mould and inflated by pressurised air 
compressor until it takes the shape of the inside of the mould to form the final product (plastic bottle) (Formlabs, 2018). 


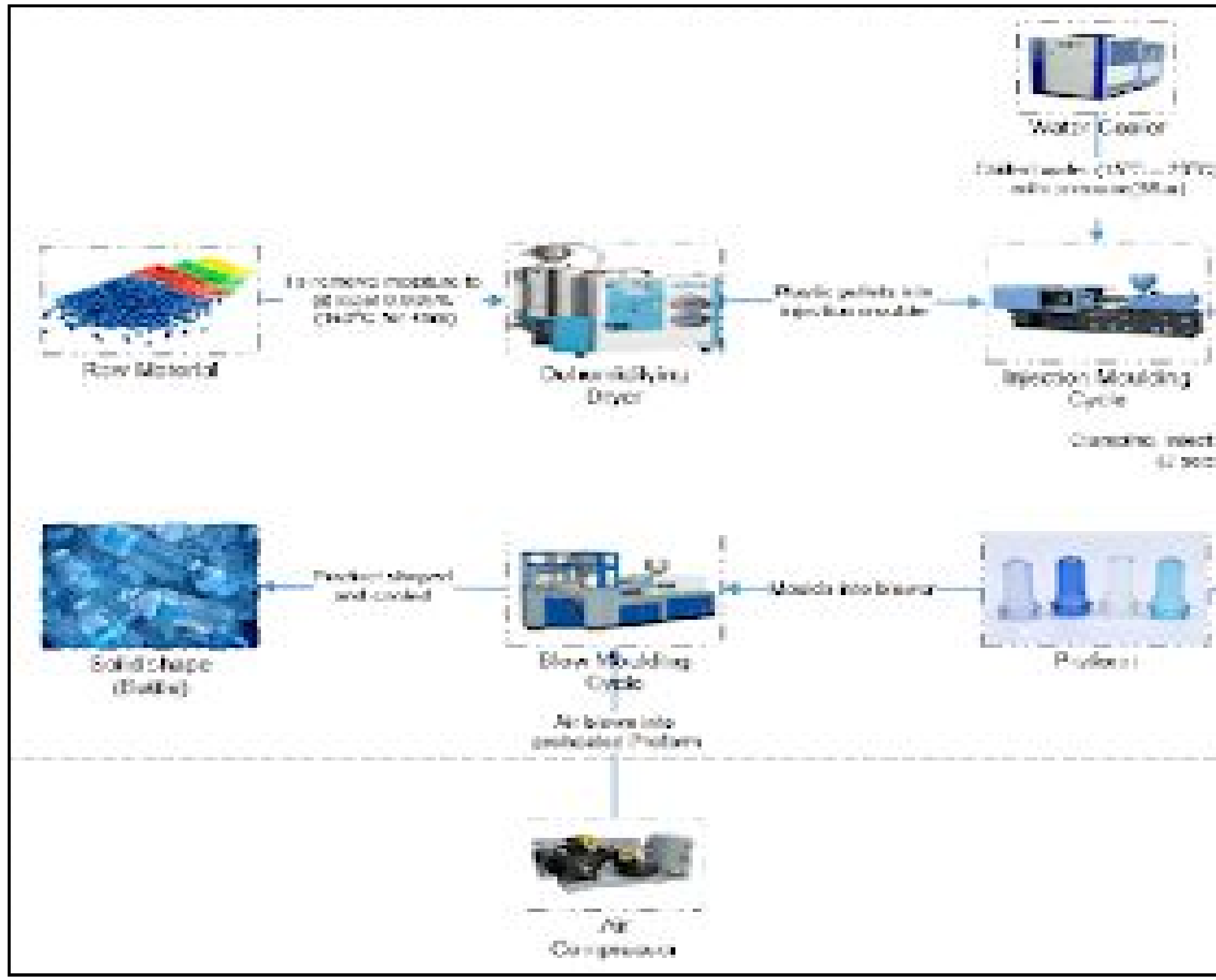

Figure 2: Two Step Production of Virgin Plastic [Polyethylene terephthalate (PET)] 


\subsection{Incorporating recycled content into plastics production}

\subsubsection{Rationale for incorporating recycled content}

The recent drive towards a circular economy approach to dealing with plastic waste has focused on reduction, reuse, composting, recycling and energy recovery. WRAP notes that because of increasing concern and awareness by consumers of the environmental impact of packaging, retailers are looking at recycling content in packaging as part of the solution. This was corroborated in an interview with the packaging manager of the supermarket chain we are currently working with on the plastics project, who acknowledged that the key motivator for their plastics reduction strategy was feedback from their customers. A 2006 WRAP project with companies like Marks and Spencer (M\&S) and Boots in collaboration with, Closed Loop London, a plastics recycling specialist, demonstrated recycled PET (rPET) could be used successfully in the production of new retail packaging. The trials were the first in the UK to demonstrate the use of significant amounts of post-consumer material in manufacturing PET packaging. For instance, $M$ \& S developed a new thermoformed bowl with 50\% rPET recycled content for its pre-paid salads range and a blow moulded bottle with 30\% rPET for non-carbonated juices and smoothies. Similarly, Boots trialled 30\% rPET across its 'Ingredients' shampoo and conditioner range with the feedback from its customers showing immense support for the new packaging (Waste \& Resources Action Programme [WRAP] 2006).

There is increasing interest from major industry players to promote circular economy through post-consumer recycled (PCR) content by boosting the demand for recycled plastics by purchasing post-consumer resin. More importantly companies aim to communicate to customers their commitment to Corporate Social Responsibility (CSR) and the environment (Waste \& Resources Action Programme [WRAP] 2006). Sustainable packaging expert Jessica Edington notes that with the current ban from China, the most critical and impactful initiative companies can take is to commit to using post-consumer resin where possible (Dreizen, 2017). For instance, PepsiCo under its "Global Sustainable Packaging Policy" increased the use of rPET by $4 \%$ to $£ 139$ million annually in 2015. Similarly, PepsiCo's Naked Juice brand uses square bottles made from $100 \%$ PCR thereby saving $25 \%$ of the energy typically used in 
conventional manufacturing processes by not using virgin PET feedstock (PEPSICO, 2016).

Economic factors are also seen as key determinants of the viability of thermoplastics recycling namely, the price of the recycled polymer and the cost of recycling must compare favourably to those of the virgin polymer and alternative forms of acceptable disposal respectively (Hopewell et al., 2009). A 2014 WRAP study points out the business opportunity arising from recycled polymers due to the significant cost saving benefit of using recycled content as they do not suffer from fluctuation in prices like virgin polymers whose cost is dependent on the cost of crude oil (Waste \& Resources Action Programme [WRAP] 2014). The literature notes that historically, landfill costs vary considerably among regions based on the underlying geology and land-use patterns which can influence the viability of recycling as an alternative disposal route. For instance, whereas the high cost of landfill is in Japan is due to the hard underlying volcanic rock, in the Netherlands, it is due to the porousness of the land caused by the sea. Thus, this high costs serve as an economic incentive to pursue either recycling or economic recovery. Similarly, economies of scale can be achieved from used waste collection from households in areas with high population density and in the UK, the introduction of kerbside collection resulted in significant increase in collection of plastic waste stream (Hopewell et al., 2009). Key local government milestones within the Resources and Waste Strategy for England (RWS) include: ensuring a consistent set of recyclable materials is collected from all households and businesses in early 2019; $50 \%$ recycling rate for household rate in 2020 and $75 \%$ recycling rate for packaging by 2030 .

\subsubsection{Implications for adding recycled content to virgin resin}

This will be explored through the challenges and opportunities associate with using recycled content. An expert in plastics, John Bozzelli explains that it is essential to ensure resins such as nylon, polycarbonate, poly (butadieneterephthalate) [PBT] and poly (ethyleneterephthalate) [PET] are dried properly before initial processing. Failure to do so will lead to the resins undergoing hydrolysis in the barrel of the moulding machine and the resultant chemical reaction significantly lowers the 
polymer chain length and causes degradation. Additionally, In terms of the appropriate ratio of recycled content to virgin, the moulding community targets 20 to 25 percent or less for blending regrind into virgin resin as blending degraded regrind into virgin at 25 percent levels may significantly alter subsequent part performance and function. Hopewell et al. (2009) argues that a major challenge of producing recycled resins is lack of compatibility between different plastic types and the associated contamination introduced during the recycling process that may be difficult to detect. For instance, a PVC recycle stream contaminated by PET will result in solid lumps of undispersed crystalline PET thereby significantly reducing the value of the recycled material and quality of the final product.

Variability related to products affect every company in one way or another as it can affect feedstock/raw materials, cause problems with the manufacturing processes and ultimately affect a customer's experience of the quality of a product (Darnbrough, 2016). Incorporating recycled resins may lead to lack of stability in the different virgin/recycle mix combinations from batch to batch which may result in temperature differences and inconsistency in the flow of the melted plastics. There is the need therefore to monitor temperature as processing the virgin resin at higher-than-recommended temperatures is a definite way of accelerating polymer degradation (Hopewell et al., 2009). Similarly, there is the increased risk of contamination originating from foreign matter particulate from the recycling process, which may present issues like variations in temperature and ease of flow of melted plastics together with clogs in the extrusion components. In addition, recycled feedstock may result in products that suffer from material variability (strong today, weak tomorrow and vice versa) because it is an extremely difficult task achieving consistency in all batches. Ultimately, the end use of the produced part will motivate the choice of recycled versus virgin plastic.

Another key challenge for the plastics recycling sector is effectively recycling mixed plastics waste. This requires an expansion of current post-consumer plastic packaging collection to capture a wider range of materials and pack types by increasing the scope of recycling to cover a larger proportion of the plastic waste 
stream. One way of tackling this is for the product design process to take into consideration its recyclability (Hopewell et al., 2009). Some argue the challenge is ensuring the end product is more valuable than the cost incurred and more importantly the value should be competitive when compared to virgin material. This can be achieved by having clean (not contaminated with non-plastic material debris and dirt from post-consumer waste) and pure (from single type of debris) product that has functional properties. Thus, the challenge is either to source used, clean, single types of plastic or cleaning and sorting mixed plastics into non contaminated single plastic streams for recycling. A 2007 UK Local Government Association (LCA) study found that about $21-40 \%$ of the volume of packaging in a regular shopping basket cannot be effectively recycled even if they are able to collect them (Local Government Association [UK] 2007). Shaxson, 2009 note if policies to promote the use of environmental design principles by industry are widely implemented, this could have a large impact on recycling performance by increasing the proportion of packaging that can economically be collected and diverted from landfill. In the 2018 RWS for England, the key issues outlined by government include: long term ambition to move away from weight-based recycling targets; improving recycling rates by ensuring consistent set of dry recyclable materials is collected from all households and businesses; ensuring businesses present recycling and food waste separately from residual waste for collection; improving urban recycling rates and ensuring Household Waste Recycling Centres are delivering value for money. Similarly, some specific strategic ambitions to be delivered are that all packaging placed on the market be recyclable, reusable and compostable by 2025; eliminate avoidable plastic waste over the lifetime of the 25 Year Environment Plan as well as to double resource productivity and eliminate avoidable waste of all kinds by 2025 .

There has been increasing public support for manufacturers, industry and government to promote sustainable production and consumption. Consumer behaviour studies involving people's preferences indicate a significant but not overwhelming proportion of people take into consideration environmental values in their purchasing patterns. For such people, confirmation of the recycled content and suitability of packaging for recycling will be a positive boost whereas an exaggeration 
of the recyclability can reduce consumer confidence. A 2015 WRAP survey show high self-reported recycling levels and in terms of capture and quality, $25 \%$ of households are completely effective recyclers. Government policies such as UK government backed WRAP Plastics Pact and EU Directive on packaging and packaging waste $(94 / 62 / E C)$ have been used to promote post-consumer recycling. Extended producer responsibility in the UK in the past include scheme for dealing in packaging recovery notes, landfill levy and presently deposit return scheme proposed by DEFRA (Waste \& Resources Action Programme [2015]).

\subsection{Plastic production within an enhanced Al/DB interface framework using virgin-recycled resin mix}

This section examines the role of an intelligent database within a simultaneous DSC-TGA thermal analysis system in promoting circular economy by using increased recycled content (15-20\%) in the plastics production process (see Figure 3). The proposed enhanced AI/DB interface framework has capability to undertake simultaneous and complementary quality control checks and material stability tests more effectively through a powerful and efficient interface that makes use of existing $\mathrm{Al}$ and DB technologies as well as future advancements in them. This is done by storing, processing and analysing historical and real time information on different tests of virgin-recycled mix specimens, quality control/quality assurance and material development measurements using adequate visualisation and analytic tools.

The DSC analytical tool is responsible for quality control of the combined virgin-recycled raw material by allowing the polymer type of the granule to be verified by accurately comparing important measurement results (like melting, glass transition temperature and recrystallization effects in the range of up to $300{ }^{\circ} \mathrm{C}$ ) to historical and real-time database polymer sample entries in the enhanced Al/DB interface [like information on literature, individual measurement graphes and statistical groupings of polymers] (Fueglein and Kaisersberger, 2015). With this type of technology, the plastic producer in accordance with quality control standard (ISO 11357 ) is able to access valuable information by querying the online library 
database. Bhatia et al. (2013) note that any performance and under-utilisation issues associated with the database management system (DBMS) is addressed through the optimisation of the functionality of the interface itself and if necessary, enhancing either the Al system or the DBMS. The intelligent database also serves as an archive by allowing information relating to literature and measurements to be added to the inbuilt libraries as well as making room for specific measurement and information to be retrieved from the database through alphanumeric filtering (Schindler et al., 2016).

Basu (2018) observes that to achieve the optimum performance and properties for a specific plastic application, extensive analysis and characterisation is required as well as continuous monitoring of the quality of the product. This poses more challenges where recycling content is involved, the biggest obstacle being controlling the quality of the feedstock. The consequences of this is most product manufacturers reluctance to use recycled feedstock due to differences in quality across batches which can have serious consequences on preserving the standard of the final-products (NETZSCH-Gerätebau $\mathrm{GmbH}, 2019$ ). Hence, the German instrumentation company argues that being able to effectively control the quality of recycled plastics is an important part of the production process of many companies as they have to be in a position to monitor, detect and identify impurities of recycled materials rapidly and in a cost-effective way. In this regard, the unique inbuilt tool allows advanced material analysis through automatic detection of impurities in the recycled plastic material by examining the spike in the measurement curve (melting effect) of the granule against similar predefined and real time measurement/identification criteria (such as another polymer type) in the AI/DB system. Similarly, the high quality time series capability of the system makes it possible to model, simulate and predict the material properties with significant success rate (Chen et al., 2020). The final stage involves identifying the type of impurities contaminating the recycled granule by comparing it with information stored in the enhanced database (measurement curve that best matches the measured peak) and has capability to identify the exact impurity correctly. 
A TGA system is very useful in investigating the thermal stability and composition of polymers by monitoring the rate and magnitude of weight change of the plastic material during the production process. An important step involves evaluating the mass of the plastic material during the injection moulding and blowing process as it undergoes changes from pellet to melted and back to solid preform before undergoing blowing into the finished product. Within a desired temperature range, we would expect the plastic material to exhibit negligible or no change in its mass. The enhanced AI/DB interface enables the inclusion of TGA data and specific heat capacity within the same database which is crucial in monitoring the temperature beyond which the polymer will melt by yielding critical and valuable information on the composition and thermal stability of the plastic pellets as they go through the injection and blowing process. For instance, NETZSCH database is capable of supporting over 1000 entries from various polymer types (Schindler et al., 2016). Matching the attributes (such as density) of a product with a sensor can be done continuously in real time and used to measure changing values of different virgin-recycled polymer mix combinations. This may require automation of part/all of the production process as a way to measure and control some aspect of quality. The TGA system tools meet instrumentation (ISO 11358) and application (ASTM D6370) standards and measurements undertaken include mass changes and temperature stability measurements ranging from $30{ }^{\circ} \mathrm{C}-1000{ }^{\circ} \mathrm{C}$ at a heating rate of $20 \mathrm{~K} /$ minute, estimation of product lifetime, moisture and volatiles content as well as composition analysis of multi-component plastic materials/blends. 


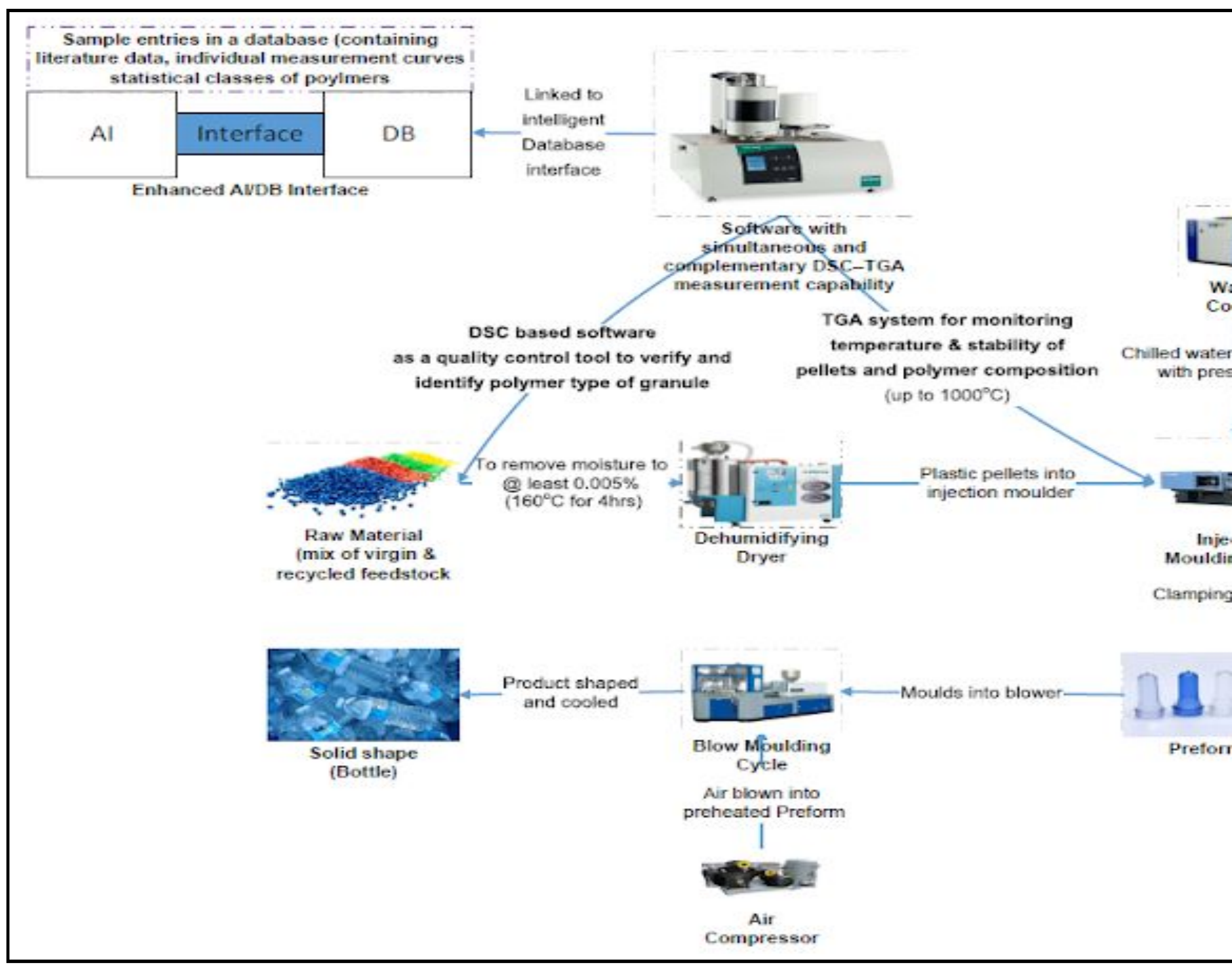

Figure 3: Plastic production incorporating intelligent database interface into a joint DSC-TGA system 
Figure 4 shows the detailed design architecture of the integration of Artificial Intelligence $(\mathrm{Al})$ and Database (DB) technologies into the joint DSC-TGA thermal analysis system with capability for storing, processing and analysing historical as well as real time information/data (Bhatia et al., 2013). The large database library and real time capability capture different virgin-recycled resin mixing ratios based on analysis of thermal properties, mechanical properties and structure (morphology) of different resin specimens to serve as a reference point for production engineers in arriving at desired check and test conditions. This enhanced database comes equipped with advanced analytics techniques for evaluating different virgin-recycled resin mix types based on testing and statistical analysis results (temperature, pressure and melt flow) of polymer properties and the impact of different mixing ratios on the material's structure using visualisation tools like charts and graphs. This information will be very useful and handy especially when a product manufacturer uses different batches of recycled resin or switches a supplier. This is essential as each batch or various suppliers of recycled resin are bound to have different consistency and stability issues meaning a feasible baseline quality control test/check regime will speed up the process ultimately leading to cost savings by minimising product defects, improving product quality and boosting efficiency of the whole plastic production process. The Al predictive and forecasting functionality ensures that production engineers are able to use time series data captured by the system to carry out predictive analytics. This will enable a company to undertake trend analysis involving looking out for patterns in quality control test results and stability check measurements and forecasting the future horizon of polymer material constituents and components with significant accuracy to stay efficient and competitive. 


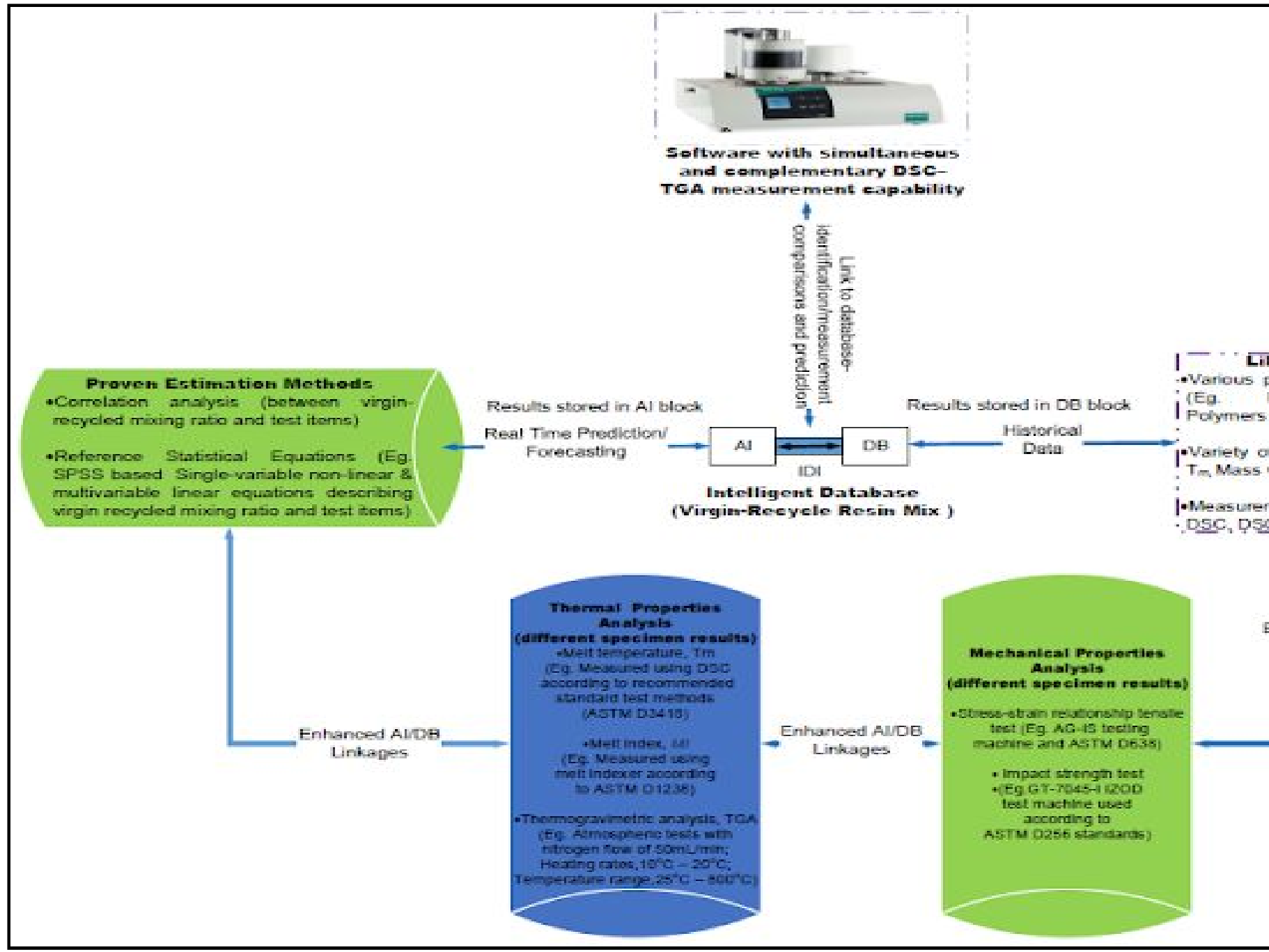

Figure 4: Enhanced AI/DB Interface and Joint DSC/TGA Software for Virgin-Recycled Resin Mix Production 


\section{Conclusion}

This paper has highlighted the growing plastic waste problem (Alimba and Faggio, 2019; Thompson et al., 2009; Parker, 2019; Chen and Yan, 2019) and the need for circular economy based sustainable ways (reduction, reuse, composting, recycling and energy recovery) to handle the waste problem (PlasticsEurope, 2019; Moraga et al., 2019) given its proven negative impact on marine life and the environment. We outlined various initiatives involving businesses, Governments, Non-Governmental Organisations (NGOs) and others working together to achieve the common vision of a circular economy for plastics in which it never becomes waste.

We pinpointed the potential problems such as lack of stability associated with different combinations of virgin/recycle polymers from batch to batch, contamination from foreign matter particulates associated with combining recycled feedstock with virgin resin in the plastic production process. As a result, plastic manufacturers resort to performing several experiments and tests to arrive at ideal quality control, temperature and melt flow conditions needed to achieve the required product consistency. We proposed a framework for the integration of Artificial Intelligence (AI) and Database (DB) technologies and Differential Scanning Calorimetry (DSC) and Thermal gravimetric analysis (TGA) techniques into the circular economy plastic production process within a joint DSC-TGA software/system with simultaneous measurement capabilities for quality control/quality assurance and monitoring of polymer composition and thermal stability of pellets.

\subsection{Main contribution to plastic production}

For our contribution, we proposed a framework for the integration of an enhanced AI/DB interface with historical and real time information/data analysis capability (Bhatia et al., 2013) into a joint DSC-TGA system. This allows for simultaneous quality control checks and thermal stability tests to be carried out during the plastic production process using different virgin-recycled resin mixing ratios, based on proven estimation methods, testing and statistical analysis results of different resin specimens using visualisation tools like charts/graphs and trend analysis through econometric forecasts. 
In this regard, this paper adds to the current topical issue of circular economy by departing from linear production model (produce-use-dispose) involving the use of only virgin raw material by embracing sustainable ways (recycled content) into the production process (Chen and Yan, 2019). The information on the thermal properties, mechanical properties and structure (morphology) of different resin specimens available through the system will serve as a reference point for production engineers in arriving at desired quality control check and stability test conditions.

\subsection{Managerial implications}

In terms of managerial implications, it will help eliminate the need to reinvent the wheel and also provide the basis for further research work on improving the plastic production methodology. For instance the large database library and real time information processing capability of the intelligent database will help manufacturers narrow down to feasible quality control/quality assurance test and stability check criteria to arrive at the ideal virgin/recycle mix. This is especially crucial when using different batches of recycled resin or when a company switches a supplier as the product in each batch or for different suppliers will have different consistency and stability issues so a baseline check/test regime will speed up the process. This will ultimately lead to cost savings, minimisation of product defects and improvement in product quality. In addition, the Al predictive function ensures production engineers are able to use time series data captured by the system to carry out trend analysis to identify patterns in quality control tests and stability measurements to forecast the future horizon of the company, which is crucial in order to remain ahead of the game.

\subsection{Limitations and Future Outlook}

The literature acknowledges an enhanced integrated AI/DB interface like the one proposed is capable of recognising measurement curves through comparison with database measurement records. However, recognition does not automatically translate into identification of a polymer (Schindler, et al., 2016). For instance, a 
limitation of a DSC database search routine is that it may lend itself to multiple interpretation of a measurement outcome, In that regard, polymer identification based on specific heat capacity may not exclusively identify a material (Schindler, et al,. 2016; 2017). Similarly, another obstacle of material identification through thermal analysis is that measurement curves may be influenced by measurement conditions which could result in the similarity value between DSC curves from the same material given different outcomes when measured with different heating rates. Another noticeable limitation of theoretical papers like this one is practical application may be difficult given the criteria for assessing quality control tests on recyclables keep changing. Similarly, achieving consistency between virgin/recycled content in real life involves lot of trial and error as different batches of the recycled material do not have the same properties. That said, there is potential under the current circular economy plastic project that inspired and funded this paper for us to undertake a case study with a local plastic production company identified for that purpose.

\section{Acknowledgement}

This paper was funded by EPSRC grant EP/S025537/1 'Evolving a circular plastics economy' to the University of Hull.

\section{References}

Adam, N., 2010. Polymer informatics. Polymer Libraries. Springler, Germany.

Agrawal, A. and Choudhary, A. (2016). Perspective: Materials informatics and big data: Realisation of the "fourth paradigm" of science in materials science. https://doi.org/10.1063/1.4946894

Alimba, C. G., Faggio, C., 2019. Microplastics in the marine environment: Current trends in environmental pollution and mechanisms of toxicological profile. Environ. Toxicol. Pharmacol. 68, 61-74. https://doi.org/10.1016/j.etap.2019.03.001

American Chemistry Council (2019). How Plastics Are Made. Available online: https://plastics.americanchemistry.com/How-Plastics-Are-Made/

[Accessed: 22/11/2019]. 
Audus, D.J., de Pablo, J.J., 2017. Polymer Informatics: Opportunities and Challenges. ACS Macro. Lett. 6, 1078-1082. https://doi.org/10.1021/acsmacrolett.7b00228.

Basu, P., 2018. Biomass Gasification, Pyrolysis and Torrefaction. Practical Design and Theory, third edition [eBook]. Elsevier Inc.

Bertino, E., Catania, B., Zarri, G.P., 2001. Intelligent Database Systems. Reading: Addison-Wesley Professional.

Bhatia, P., Khurana, N. and Sharma, N., 2013. Intuitive Approach to use Intelligent Database for Prediction. Int. J. Comp. Appl. (0975 - 8887) 83, 15. doi:10.5120/4527-2923.

British Broadcasting Corporation [BBC], 2019. War on Plastic Series. Nature and Environment Programme

Brown, N., Sandholm, T., 2017. Superhuman Al for heads-up no-limit poker: libratus beats top professionals. Science 359, 418-424. doi:10.1126/science.aao1733.

Calleja, D. (2019). Why the "New Plastics Economy" must be a circular economy. Field Actions Science Reports, Special Issue 19, 22 - 27.

Charles, R. G., Davies, M. L., Douglas, P., Hallin, I. L., 2018. Sustainable Solar Energy Collection and Storage for Rural Sub-Saharan Africa. In: Letcher, T. M., Fthenakis, V. M. (Eds.), A Comprehensive Guide to Solar Energy Systems with Special Focus on Photovoltaic Systems. Elsevier Inc, 81 - 107. https://doi.org/10.1016/B978-0-12-811479-7.00005-1

Chen, X., Yan, N., 2019. A Brief Overview of Renewable Plastics. Material Today Sustainability, 7-8 . https://doi.org/10.1016/j.mtsust.2019.100031

Chen, G., Shen, Z., Iyer, A., Ghumman, U.F., Tang, S., Bi, J., Chen, W., Li, Y., 2020. Machine Learning Assisted De Novo Design of Organic Molecules and Polymers: Opportunities and Challenges. Polymers (Basel) 12, 163. https://doi.org/10.3390/polym12010163.

Darnbrough, P., 2016. Monitoring and controlling quality in process manufacturing. Intech Magazine. (Mar-Apr).

Department for Environment, Food and Rural Affairs [DEFRA], (2018). Our Waste, Our Resources: A Strategy For England. Available online: 
https://www.gov.uk/government/publications/resources-and-waste-strategy-for-engla nd [Accessed 26/12/2019]

Dreizen, C., 2017. Sustainable Packaging: The race to increase recycled content in packaging. Packaging Digest. Available online: https://www.packagingdigest.com/sustainable-packaging/the-race-to-increase-recycl ed-content-in-packaging-2017-12-04\#comments [Accessed: 01/04/2020].

Dwivedi, C., Pandey, I., Pandey, H., Ramteke, P. W., Pandey, A. C., Mishra, S. B., and Patil, S. (2017). Nano-and Microscale Drug Delivery Systems. Design and Fabrication. Elsevier Inc.

Ellen MacArthur Foundation, 2017. The New Plastics Economy Global Commitment. Available online: https://www.newplasticseconomy.org/projects/global-commitment [Accessed 23/12/2019].

European Commission [EC] (2018). A European Strategy For Plastics In A Circular Economy. Available online: https://ec.europa.eu/environment/circular-economy/pdf/plastics-strategy-brochure.pd f [Accessed: 23/12/2019]

Formlabs (2018). Guide to Manufacturing Processes for Plastics. Available online: https://formlabs.com/blog/guide-to-manufacturing-processes-for-plastics/ [Accessed 26/12/2019].

Fortunato, A. (2013). In: Pignatello, R. (Ed.), Drug-Biomembrane Interaction Studies: The Application of Calorimetric Techniques. DSC: history, instruments and devices, Woodhead Publishing Limited, 169 - 212.

Fueglein, E., 2015. About the use of IDENTIFY-a thermoanalytical database-for characterisation and classification of recycled polyamides. J. Therm. Anal. Calorim. 121, 1353-1357. https://doi.org/10.1007/s10973-015-4583-3.

Fueglein, E., Kaisersberger, E., 2015. About the development of databases in thermal analysis. J. Therm. Anal. Calorim. 120, 23-31. https://doi.org/10.1007/s10973-014-4381-3.

García-Muiña, F.E., González-Sánchez, R., Ferrari, A.M., Settembre-Blundo, D., 2018. The Paradigms of Industry 4.0 and Circular Economy as Enabling Drivers for the Competitiveness of Businesses and Territories: The Case of an Italian Ceramic Tiles Manufacturing Company. Soc. Sci. 7(12), 255. 
Gehring, J., 2000. With radiation crosslinking of engineering plastics into the next millennium. Radiat. Phys. Chem. 57 (3-6), 361-365. https://doi.org/10.1016/S0969-806X(99)00405-3

Geissbauer, R., 2016. Industry 4.0: Building the digital enterprise. 2016 Global Industry $\quad 4.0 \quad$ Survey. PWC. Available online: https://www.pwc.com/gx/en/industries/industries-4.0/landing-page/industry-4.0-buildi ng-your-digital-enterprise-april-2016.pdf [Accessed 09/09/2019].

Gil, Y., Greaves M., Hendler, J., Hirsh, H., 2014. Amplify scientific discovery with artificial intelligence. Science 346, 171-172. http://doi:10.1126/science.1259439.

He, K., Zhang, X., Ren, S. and Sun, J. (2016). Deep residual learning for image recognition. In Proc. IEEE Computer Society Conference on Computer Vision and Pattern Recognition. 770-778. IEEE, USA: Las Vegas, NV.

Hopewell, J., Dvorak, R., Kosior, E., 2009. Plastics recycling: challenges and opportunities. Philos. Trans. R Soc. Lond B Biol. Sci., 364 (1526), 2115-2126. https://doi.org/10.1098/rstb.2008.0311.

Kalmykova, Y., Sadagopan, M. and Rosado, L. (2018). Circular Economy - From review of theorities and practices to development of implementation tools. Resource, Conservation and Recycling. 135, pp. 190 - 201. https://doi.org/10.1016/j.resconrec.2017.10.034.

Liu, X., Yu, W., 2006. Evaluating the Thermal Stability of High Performance Fibers by TGA. J. Appl. Polym. Sci. 99 (3), 937-944. https://doi.org/10.1002/app.22305.

Local Government Association [UK] (2007). War on waste: food packaging study. UK:Local Government Authority.

Lowe, R., 2017. Thermal Analysis in Plastics: Thermogravimetric Analysis. Impact Solutions. https://www.impact-solutions.co.uk/thermal-analysis-plastics-tgal [ Accessed: 24/12/2019]

Ma, Z. (Ed.), (2007). Intelligent databases: Technologies and Applications. IGI Global.

Majewsky, M., Bitter, H., Eiche, E., Horn, H., 2016. Determination of microplastic polyethylene (PE) and polypropylene (PP) in environmental samples using thermal 
analysis (TGA-DSC). Science of The Total Environment, 568, 507-511. doi: 10.1016/j.scitotenv.2016.06.017.

Moraga, G., Huysveld, S., Mathieux, F., Blengini, G. A., Alaerts, L., Acker, K. V., De Meester, S., Dewulf, J. (2019). Circular Economy Indicators: What do they measure. Resour. Conserv. Recycl. 146, 452-461.

Mrowiec, B. (2018). Plastics in the circular economy (CE). Environmental Protection and Natural Resources. The Journal of Institute of Environmental Protection-National Research Institute, 29(4): pp. 16-19. doi:https://doi.org/10.2478/oszn-2018-0017.

NETZSCH-Gerätebau GmbH, 2019. How DSC Can Control the Quality of Recycled Plastic Materials. AZoM. Available online: https://www.azom.com/article.aspx?ArticlelD=18717 [Accessed 23/12/2019].

Netzsch Group, 2019. Thermal Characterisation of Polymers. Analyzing \& Testing Unit.

Nihalani, N., Silakari, S., Motwani, M., 2009. Integration of Artificial Intelligence and Database Management System: An Inventive Approach for Intelligent Databases, 2009 First International Conference on Computational Intelligence, Communication Systems and Networks. IEEE, indore, pp 35-40. https://doi.org/10.1109/CICSYN.2009.54.

Opsomer, R., Pennington, J., 2016. What are the drawbacks of today's plastics economy? World Economy Forum.

Parker, L. (2019). The world's plastic pollution crisis explained. National Geographic. Available online: https://www.nationalgeographic.com/environment/habitats/plastic-pollution/

[Accessed: 26/12/2019]

Parsaye, K., Chingnell, M., Khoshafian, S. and Wong, H., 1989. IntelligentDatabases. Wiley.

Patterson \& Rothwell (2019). Plastic Injection Moulding Explained. Injection Moulding Specialists. Available online: https://www.patterson-rothwell.co.uk/plastic-injection-moulding-explained/ [Accessed 26/12/2019]. 
Payne, J., McKeown, P. and Jones, M. D., 2019. A circular economy approach to plastic waste. Polymer Degradation and Stability, 165, 170-181. https://doi.org/10.1016/j.polymdegradstab.2019.05.014.

PEPSICO, 2016. Global Sustainable Packaging Policy. Available online: https://www.pepsico.com/docs/album/esg-topics-policies/pepsico-sustainable-packa ging-policy.pdf?sfvrsn=d808643c_4 [Accessed: 01/04/2020].

PlasticEurope, 2019. How Plastics Are Made. Association of Plastics Manufacturers. Available online: https://www.plasticseurope.org/en/about-plastics/what-are-plastics/how-plastics-aremade [Accessed: 22/11/2019].

PlasticsEurope (2019). Plastics's Contribution To The Circular Economy. Available online: https://www.plasticseurope.org/en/focus-areas/circular-economy [Accessed 23/12/2019].

Plastics Industry Association [PLASTICS] (1988). Classification of Plastics.

Popenici, S.A.D., Kerr, S., 2017. Exploring the impact of artificial intelligence on teaching and learning in higher education. RPTEL 12, 22. https://doi.org/10.1186/s41039-017-0062-8.

Rajan, K., 2013. Informatics for Materials Science and Engineering: Data-driven Discovery for Accelerated Experimentation and Application. Butterworth-Heinemann, UK.

Sarkisov, L., Kim, J., 2015. Computational structure characterisation tools for the era of material informatics. Chem. Eng. Sci. 121, 322-330. https://doi.org/10.1016/j.ces.2014.07.022.

Sastri, V.R., 2010. Plastics in Medical Devices: properties, requirements and applications. Plastics Design Library [eBook]. Elsevier Inc.

Schick, C., 2009. Differential scanning calorimetry (DSC) of semicrystalline polymers. Anal Bioanal Chem 395:1589-1611. doi:10.1007/s00216-009-3169-y.

Schindler, A., Strasser, C., Schmölzer, S., Bodek, M., Seniuta R,. Wang, X., 2016. Database-supported thermal analysis involving automatic evaluation, identification and classification of measurement curves. J. Therm. Anal. Calorim. 123, 2405-2414. https://doi.org/10.1007/s10973-015-5026-x 
Schindler, A., Doedt, M., Gezgin, Ş., Menzel, J., Schmozer, S., 2017. Identification of polymers by means of DSC, TG, STA and computer-assisted database search. $J$ Therm Anal Calorim 129, 833-842. https://doi.org/10.1007/s10973-017-6208-5

Sciencing.com, 2018. The Plastic Manufacturing Process. Available online: https://sciencing.com/how-does-4963354-plastic-manufacturing-process.html [Accessed 26/12/2019].

Shaxson, L., 2009. Structuring policy problems for plastics, the environment and human health: reflections from the UK. Phil. Trans. R. Soc. B, 364, 2141-2151. doi:10.1098/rstb.2008.0283.

Silver, D., Huang, A., Maddison, C., Guez, A., Sifre, L., van den Driessche, G., Schrittwieser, J., Antonoglou, I., Panneershelvam, V., Lanctot, M., Dieleman, S., Grewe, D., Nham, J., Kalchbrenner, N., Sutskever, I., Lillicrap, T., Leach, M., Kavukcuoglu, K., Graepel, T., Hassabis, D., 2016. Mastering the game of Go with deep neural networks and tree search. Nature 529, 484-489. https://doi.org/10.1038/nature16961.

Silver, D., Schrittwieser, J., Simonyan, K. Antonoglou, I., Huang, A., Guez, A., Hubert, T., Baker, L., Lai, M., Bolton, A., Chen, Y., Lillicrap, T., Hui, F., Sifre, L., van den Driessche, G., Graepel, T., Hassabis, D., 2017. Mastering the game of Go without human knowledge. Nature 550, 354-359. https://doi.org/10.1038/nature24270.

Thompson, R.C., Moore, C.J., von Saal, F.S., Swanna, S.H., 2009. Plastics, the environment and human health: current consensus and future trends. Phil. Trans. R. Soc B, 364 (1526), 2153-2166. https://doi.org/10.1098/rstb.2009.0053.

UKRI/EPSRC (2018). Creative Circular Economy Approaches to Eliminate Plastics Waste. Available online: https://epsrc.ukri.org/files/funding/calls/2018/circular-plastics-call-document/ [Accessed 23/12/2019]

United Nation [UN], 2012. 10-Year Framework of Programmes on Sustainable Consumption and Production (10YFP). United Nations Environment Programme.

UN International Basel Convention Treaty (2018). Basel Convention on the Control of Transboundary Movements of Hazardous Waste and their Disposal. Available online:

http://www.basel.int/TheConvention/Overview/TextoftheConvention/tabid/1275/Defau It.aspx [Accessed 23/12/2019] 
Waste \& Resources Action Programme [WRAP] (2006). Large-scale demonstration of viability of recycled PET (rPET) in retail packaging. In collaboration with Closed Loop London, recycling specialists.

Waste \& Resources Action Programme [WRAP] (2014). Using recycled polymers in new products: A business opportunity.

Waste \& Resources Action Programme [WRAP] (2015). 3Rs recycling knowledge, attitudes and reported behaviour survey. Prepared by Phil Downing, Roisin Jones and Alex Plumb.

Waste \& Resources Action Programme [WRAP] (2018). The UK Plastic Pact. Launched in collaboration with Ellen MacArthur Foundation and supported by Defra.

Wong, C. (2010). A Study of Plastic Recycling Supply Chain. A research project sponsored by The Chartered Institute of Logistics and Transport (UK). Seed Corn Research Fund 2009-2010.

World Economic Forum, 2016. The New Plastics Economy: Rethinking the future of plastics. In collaboration with the Ellen MacArthur Foundation. Available online: http://www3.weforum.org/docs/WEF_The_New_Plastics_Economy.pdf [Accessed 23/12/2019]

Wu, S., Kondo, Y., Kakimoto, M., Yang, B., Yamada, H., Kuwajima, I., Lambard, G., Hongo, K., Xu, Y., Shiomi, J., Schick, C., Morikawa, J., Yoshida, R., 2019. Machine-learning-assisted discovery of polymers with high thermal conductivity using a molecular design algorithm npj. Comput. Mater. 5, 66. https://doi.org/10.1038/s41524-019-0203-2. 